\title{
Robust decoupled visual servoing based on structured light
}

\author{
Jordi Pagès ${ }^{\dagger}$ Christophe Collewet François Chaumette $^{\S}$ Joaquim Salvi ${ }^{\dagger}$ \\ † Institut d'Informàtica i Aplicacions \\ University of Girona \\ Girona, Spain \\ $\ddagger$ Cemagref \\ 17 Avenue de Cucillé \\ Rennes, France \\ $\S$ IRISA / INRIA Rennes \\ Campus de Beaulieu \\ Rennes, France
}

\begin{abstract}
This paper focuses on the problem of realizing a plane-to-plane virtual link between a camera attached to the end-effector of a robot and a planar object. In order to do the system independent to the object surface appearance, a structured light emitter is linked to the camera so that 4 laser pointers are projected onto the object. In a previous paper we showed that such a system has good performance and nice characteristics like partial decoupling near the desired state and robustness against misalignment of the emitter and the camera [10]. However, no analytical results concerning the global asymptotic stability of the system were obtained due to the high complexity of the visual features utilized. In this work we present a better set of visual features which improves the properties of the features in [10] and for which it is possible to prove the global asymptotic stability.
\end{abstract}

Index Terms - Visual servoing, stability analysis, robustness, structured light.

\section{INTRODUCTION}

Image-based eye-in-hand visual servoing [5] is a robot control technique based on visual features extracted from the image acquired by a camera attached to the robot end-effector. The goal consists of moving the robot to a desired position where the visual features contained in a $k$-dimensional vector $\mathbf{s}$ become $\mathbf{s}^{*}$. The velocity of the features $\dot{\mathbf{s}}$ is related to the relative camera-object motion according to the well-known equation

$$
\dot{\mathbf{s}}=\mathbf{L}_{\mathbf{s}} \mathbf{v}
$$

where $\mathbf{L}_{\mathbf{s}}$ is the so-called interaction matrix and $\mathbf{v}=$ $\left(V_{x}, V_{y}, V_{z}, \Omega_{x}, \Omega_{y}, \Omega_{z}\right)$ is the relative camera-object velocity (kinematic screw). This linear relationship is usually used to design a control law whose aim is to cancel the following vision-based task function

$$
\mathbf{e}=\mathbf{C}\left(\mathbf{s}-\mathbf{s}^{*}\right)
$$

where $\mathbf{C}$ is a combination matrix chosen as $\mathbf{I}_{n}$ when $n=k$, $n$ being the number of controlled axes (hereafter we assume this choice). Then, by imposing an exponential decrease of the task function a simple proportional control law can be synthesized

$$
\mathbf{v}=-\lambda \widehat{\mathbf{L}}_{\mathbf{s}}^{+} \mathbf{e}
$$

with $\lambda$ a positive gain and $\widehat{\mathbf{L}_{\mathbf{s}}}$ a model of the interaction matrix which is usually chosen as its value in the desired state (noted as $\mathbf{L}_{\mathbf{s}}^{*}$ ) [4].

During the last years, an important part of the visual servoing research has been focused on investigating approaches enlarging the convergence domain, which ensure that the camera will reach the desired position even if the initial state is quite far from the desired one [1], [9], [12]. A necessary condition for this is that the interaction matrix must be nonsingular. A design strategy which can avoid singularities of $\mathbf{L}_{\mathbf{s}}$ is to obtain decoupled visual features, so that each one only controls one degree of freedom (dof). Even if such control design seems to be out of reach, there are several works concerning the problem of partially decoupling dofs. Hybrid techniques are based on controlling rotational dof in the cartesian space while the translational ones are controlled by image information [3], [9]. However, they require partial pose estimation of the object at each iteration. On the other hand, some pure image-based techniques have succeed to decouple rotational dof from translational ones near the desired state [2], [13]. Concerning the stability analysis, most part of techniques for which it has been possible to find analytical conditions are hybrid approaches like in [1], [9] or more recently, the extended-2D visual servoing [12]. Usually, the global asymptotic stability analysis of pure image-based techniques is too complex even in absence of calibration errors.

Another important research topic in image-based visual servoing is to improve the camera trajectory in the cartesian space. It is well known that even if an exponential decrease on the task function is achieved, it does not imply a suitable camera trajectory. This is mainly due to strong non-linearities in the interaction matrix. Important efforts have been done in order to improve the mapping from the feature space to the camera velocities [8], [13].

In this paper we propose an image-based visual servoing approach based on visual features provided by a structured light emitter attached to the camera. The goal of our task consists on fixing a plane-to-plane virtual link between the camera and a planar object. A first advantage of using structured light is that the image processing is highly simplified and the application becomes independent to the object appearance. Furthermore, the projected pattern can be chosen in order to produce a suitable set of visual features in the image which optimizes the interaction matrix and contributes to a better 3D trajectory. Some works combining visual servoing and structured light can be found in [1], [6], [7]. The main contribution of our approach is that the desirable characteristics previously mentioned are achieved in the ideal case of a calibrated system. Concretely, the interaction matrix 
decouples rotational dof from translational ones in all the workspace. This nice structure of the interaction matrix allows the global asymptotic stability to be proven when using two different control laws based on (3). Furthermore, one of the control laws generates monotonic camera velocities producing suitable camera 3D trajectories which can be easily accomplished by the robot. Moreover, the local asymptotic stability is also proved in presence of large calibration errors.

The paper is structured as follows. First, in Section II the architecture and model of our approach is presented. Secondly, a new set of visual features which achieve decoupling in all the workspace is presented in Section III. Afterwards, the global asymptotic stability of the system is developed in Section IV. In Section V a simple transformation of the proposed visual features is explained in order to increase the convergence domain in front of laser-cross misalignment. Stability analysis taking into account misalignments is presented in Section VI. Some experiments validating the analytical results are shown in Section VII. Finally, conclusions are discussed in Section VIII.

\section{SYSTEM MODELLING}

Our system is composed of a camera and a structured light emitter both attached to the end-effector of a robot manipulator. The structured light emitter is composed of 4 laser pointers and has been modelled assuming that all the lasers have the same direction and that they are symmetrically distributed with respect to the center of the laser-cross frame $\{L\}$ (see Fig. 1a). In this model the camera frame coincides with the laser-cross frame as in Fig. 1b. In Section VI we will see that the system is robust even if these modelling assumptions are violated.

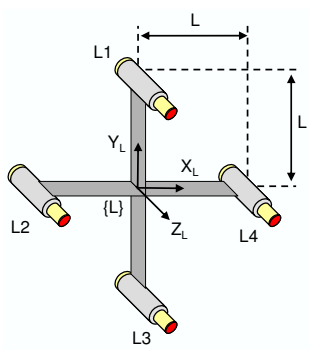

a)

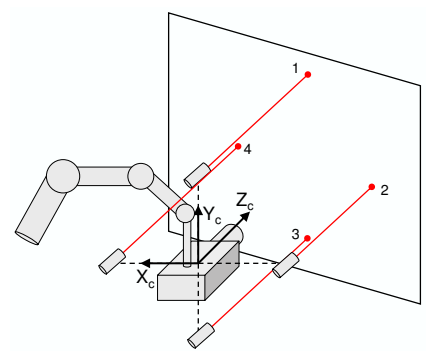

b)
Fig. 1. a) Scheme of the structured light emitter. b) Ideal linking of the camera and the laser-cross.

We face the problem of designing a robust image-based visual servoing approach to fulfill the plane-to-plane positioning task. Such a task requires to control only 3 combinations of degrees of freedom, so that a set of 3 independent visual features is enough. Then, the task function is defined as $\mathbf{e}=\mathbf{s}-\mathbf{s}^{*}$. Let model the planar object by the equation

$$
\underline{\mathbf{n}}^{\top} \mathbf{X}+D=0
$$

being $\mathbf{n}=(A, B, C)$ the unitary normal vector to the plane and $D$ its distance to the origin of the camera frame. We take as convention $C>0$ which implies that $D<0$. Then, the interaction matrix of the point projected by a laser pointer attached to the camera is [10]

$$
\mathbf{L}_{\mathbf{x}}=\frac{1}{\Pi_{0}}\left(\begin{array}{cccccc}
\frac{-A X_{0}}{Z} & \frac{-B X_{0}}{Z} & \frac{-C X_{0}}{Z} & X_{0} \varepsilon_{1} & X_{0} \varepsilon_{2} & X_{0} \varepsilon_{3} \\
\frac{-A Y_{0}}{Z} & \frac{-B Y_{0}}{Z} & \frac{-C Y_{0}}{Z} & Y_{0} \varepsilon_{1} & Y_{0} \varepsilon_{2} & Y_{0} \varepsilon_{3}
\end{array}\right)
$$

with

$$
\begin{aligned}
\Pi_{0} & =\underline{\mathbf{n}}^{\top}\left(\mathbf{X}_{0}-\mathbf{x} Z\right) \\
\left(\varepsilon_{1}, \varepsilon_{2}, \varepsilon_{3}\right) & =\underline{\mathbf{n}} \times \mathbf{x}
\end{aligned}
$$

where $\mathbf{x}=(x, y, 1)=(X / Z, Y / Z, 1)$ are the normalized coordinates of the projected point, and $\mathbf{X}_{0}=\left(X_{0}, Y_{0}, 0\right)$ is the origin of the laser pointer defined as the intersection of the laser pointer direction and the image plane $Z=0$.

When the laser-cross is perfectly aligned with the camera, the interaction matrices of the 4 projected points can be calculated by using the parameters presented in Table I. In our

TABLE I

IDEAL MODEL PARAMETERS

\begin{tabular}{cccccc} 
Laser & $X_{0}$ & $Y_{0}$ & $\mathrm{x}$ & $\mathrm{y}$ & $Z_{i}$ \\
\hline 1 & 0 & $L$ & 0 & $L / Z_{1}$ & $-(B L+D) / C$ \\
2 & $-L$ & 0 & $-L / Z_{2}$ & 0 & $(A L-D) / C$ \\
3 & 0 & $-L$ & 0 & $-L / Z_{3}$ & $(B L-D) / C$ \\
4 & $L$ & 0 & $L / Z_{4}$ & 0 & $-(A L+D) / C$ \\
\hline
\end{tabular}

previous work [10], a set of 3 visual features was proposed based on the area enclosed by the four points in the image and angles between virtual segments passing through these points. These features showed a local decoupling of the rotational dof $\left(\Omega_{x}\right.$ and $\left.\Omega_{y}\right)$ from the depth control $\left(V_{z}\right)$ near the desired state $\left(A=B=0, C=1\right.$ and $\left.D=-Z^{*}\right)$. However, no results about global asymptotic stability were reported due to the high complexity of the general interaction matrix. In the following section, we present a new set of visual features which allows global asymptotic stability to be demonstrated.

\section{DECOUPLING VISUAL FEATURES FOR THE ENTIRE WORKSPACE}

We look for a set of 3 visual features which decouples as much as possible the controlled degrees of freedom not only near the desired position but for any camera-object pose. Let us take a look at the interaction matrices of $y_{1}^{-1}, y_{3}^{-1}, x_{2}^{-1}$ and $x_{4}^{-1}$, noting that $\mathbf{L}_{\mathbf{s}}=\left(\begin{array}{ll}\mathbf{L}_{\mathbf{s}}^{V} & \mathbf{L}_{\mathbf{s}}^{\Omega}\end{array}\right)$

$$
\begin{aligned}
& \mathbf{L}_{y_{1}^{-1}}^{V}=\left(\begin{array}{lll}
-K_{1} & -K_{2} & -K_{3}
\end{array}\right) \\
& \mathbf{L}_{y_{1}^{-1}}^{\Omega}=\left(\begin{array}{lll}
-\frac{K_{2}(B L+D)}{C}-1 & \frac{K_{1}(B L+D)}{C} & K_{1} L
\end{array}\right) \\
& \mathbf{L}_{y_{3}^{-1}}^{V}=\left(\begin{array}{lll}
K_{1} & K_{2} & K_{3}
\end{array}\right) \\
& \mathbf{L}_{y_{3}^{-1}}^{\Omega}=\left(\begin{array}{lll}
-\frac{K_{2}(B L-D)}{C}-1 & \frac{K_{1}(B L-D)}{C} & K_{1} L
\end{array}\right) \\
& \mathbf{L}_{x_{2}^{-1}}^{V}=\left(\begin{array}{lll}
K_{1} & K_{2} & K_{3}
\end{array}\right) \\
& \mathbf{L}_{x_{2}^{-1}}^{\Omega}=\left(\begin{array}{lll}
-\frac{K_{2}(A L-D)}{C} & \frac{K_{1}(A L-D)}{C}+1 & -K_{2} L
\end{array}\right) \\
& \mathbf{L}_{x_{4}^{-1}}^{V}=\left(\begin{array}{lll}
-K_{1} & -K_{2} & -K_{3}
\end{array}\right) \\
& \mathbf{L}_{x_{4}^{-1}}^{\Omega}=\left(\begin{array}{lll}
-\frac{K_{2}(A L+D)}{C} & \frac{K_{1}(A L+D)}{C}+1 & -K_{2} L
\end{array}\right)
\end{aligned}
$$


with

$$
K_{1}=\frac{A}{L C} \quad K_{2}=\frac{B}{L C} \quad K_{3}=\frac{1}{L}
$$

It is obvious that simple combinations of such features can lead to a decoupled system. We have chosen the following set of visual features

$$
\mathbf{s}=\left(y_{1}^{-1}-y_{3}^{-1}, \quad y_{1}^{-1}+y_{3}^{-1}, \quad x_{2}^{-1}+x_{4}^{-1}\right)
$$

whose interaction matrix is

$$
\mathbf{L}_{\mathbf{s}}=\left(\begin{array}{cccccc}
-\frac{2 A}{L C} & -\frac{2 B}{L C} & -\frac{2}{L} & -\frac{2 B D}{L C^{2}} & \frac{2 A D}{L C^{2}} & 0 \\
0 & 0 & 0 & -\frac{2\left(1-A^{2}\right)}{C^{2}} & \frac{2 A B}{C^{2}} & \frac{2 A}{C} \\
0 & 0 & 0 & -\frac{2 A B}{C^{2}} & \frac{2\left(1-B^{2}\right)}{C^{2}} & -\frac{2 B}{C}
\end{array}\right)
$$

which is always rank 3 unless for degenerated cases. Note that the rotational part is decoupled from the translational one for any camera-object pose. To our knowledge, there are no other image-based approach where the interaction matrix has such a high order of decoupling for any state. Another interesting characteristic of this interaction matrix is that it can be expressed in terms of the task function components. By using the normalized image points coordinates for the ideal case (presented in Table I), the components of $\mathbf{e}=\mathbf{s}-\mathbf{s}^{*}=$ $\left(e_{1}, e_{2}, e_{3}\right)$ can be expressed as follows

$$
e_{1}=\frac{-2\left(D+C Z^{*}\right)}{L C}, e_{2}=-2 \frac{B}{C}, e_{3}=-2 \frac{A}{C}
$$

We remember that $\underline{\mathbf{n}}$ is a unitary vector so that $C=\sqrt{1-A^{2}-B^{2}}$. Thus, we have a system of 3 equations and 3 unknowns $(A, B$ and $D$ ) whose unique solution is

$$
A=-\frac{e_{3}}{h}, B=-\frac{e_{2}}{h}, D=-\frac{e_{1} L+2 Z^{*}}{h}
$$

with $h=\sqrt{e_{2}^{2}+e_{3}^{2}+4}$ and therefore $C=2 / h$. Using these equivalences, the interaction matrix can be expressed in terms of the task function components as follows

$$
\mathbf{L}_{\mathbf{s}}(\mathbf{e})=\left(\begin{array}{cccccc}
\frac{e_{3}}{L} & \frac{e_{2}}{L} & -\frac{2}{L} & -\frac{e_{2}\left(e_{1} L+2 Z^{*}\right)}{2 L} & \frac{e_{3}\left(e_{1} L+2 Z^{*}\right)}{2 L} & 0 \\
0 & 0 & 0 & -\frac{1}{2} e_{2}^{2}-2 & \frac{1}{2} e_{2} e_{3} & -e_{3} \\
0 & 0 & 0 & -\frac{1}{2} e_{2} e_{3} & 2+\frac{1}{2} e_{3}^{2} & e_{2}
\end{array}\right)
$$

This allows us to decide which model of interaction matrix is used in the control law (3). The first choice consists of a non-constant control law where an estimation of $\mathbf{L}_{\mathbf{s}}$ at each iteration is used. On the other hand, a constant control law based on $\mathbf{L}_{\mathbf{s}}^{*}$ can be used

$$
\widehat{\mathbf{L}_{\mathbf{s}}}=\mathbf{L}_{\mathbf{s}}^{*}=\left(\begin{array}{cccccc}
0 & 0 & -2 / L & 0 & 0 & 0 \\
0 & 0 & 0 & -2 & 0 & 0 \\
0 & 0 & 0 & 0 & 2 & 0
\end{array}\right)
$$

Note that this matrix does not contain any non-linearities neither depth information. Therefore, each visual feature varies proportionally to the dof which controls. Thus, if the task function has a good decreasing, suitable camera velocities will be produced [8].

\section{STABILITY ANALYSIS OF THE IDEAL SYSTEM}

In this section we present the global asymptotic stability analysis of the ideal system, that is, when the laser-cross is perfectly aligned with the camera. Given the general control law in (3) the closed-loop equation describing the dynamics of the task function in terms of time is

$$
\dot{\mathbf{e}}(t)=-\lambda \mathbf{L}_{\mathbf{s}}(\mathbf{e}(t)) \widehat{\mathbf{L}}_{\mathbf{s}}^{+} \mathbf{e}(t)
$$

As can be seen, $\mathbf{e}=\mathbf{0}$ is an equilibrium point since when $\mathbf{e}=\mathbf{0}$ then $\dot{\mathbf{e}}=\mathbf{0}$. The uniqueness of the equilibrium point is demonstrated by showing that the kernel of the product of matrices $\mathbf{M}=\mathbf{L}_{\mathrm{s}} \widehat{\mathbf{L}}_{\mathrm{s}}^{+}$is empty. In the following subsections we show that the equilibrium point is unique and globally asymptotically stable both for control laws.

\section{A. Non-constant control law}

The global asymptotic stability of the ideal system is obvious since $\mathbf{L}_{\mathrm{s}}$ can be perfectly estimated at each iteration so that $\widehat{\mathbf{L}_{\mathbf{s}}}=\mathbf{L}_{\mathbf{s}}$. Then $\mathbf{M}$ is the identity, whose kernel is empty and therefore the equilibrium point is unique, and the closed-loop equation becomes simply $\dot{\mathbf{e}}(t)=-\lambda \mathbf{e}(t)$ which ensures a pure exponential decrease of the task function. However, it is not easy to predict the camera velocities.

\section{B. Constant control law}

When using the constant diagonal matrix in (13) the determinant of $\mathrm{M}$ is $1 / C^{2}$, which is always different to 0 and therefore the equilibrium point is unique. Hereafter, we solve the differential equation in order to obtain the behavior of the task function and to demonstrate its convergence to the equilibrium point. The differential system (14) can be written as

$$
\begin{gathered}
\dot{e}_{1}(t)=-\frac{\lambda}{4 L}\left(e_{1}(t)\left(4 L+e_{2}(t)^{2} L+e_{3}(t)^{2} L\right)\right. \\
\left.+2 Z^{*}\left(e_{2}(t)^{2}+e_{3}(t)^{2}\right)\right) \\
\dot{e}_{2}(t)=-\frac{\lambda}{4}\left(e_{2}(t)^{3}+4 e_{2}(t)+e_{2}(t) e_{3}(t)^{2}\right) \\
\dot{e}_{3}(t)=-\frac{\lambda}{4}\left(e_{3}(t)^{3}+4 e_{3}(t)+e_{3}(t) e_{2}(t)^{2}\right)
\end{gathered}
$$

After some tedious developments we find the solutions [11]

$$
\begin{aligned}
& e_{1}(t)=\frac{2 e_{1}(0)}{a(t)}-\frac{2 b Z^{*} \arctan \left(\frac{b(a(t)-2)}{b^{2}+2 a(t)}\right)}{a(t) L} \\
& e_{2}(t)=2 e_{2}(0) / a(t) \\
& e_{3}(t)=2 e_{3}(0) / a(t)
\end{aligned}
$$

with

$$
\begin{aligned}
a(t) & =\sqrt{\left(e_{2}^{2}(0)+e_{3}^{2}(0)\right)\left(\exp ^{2 \lambda t}-1\right)+4 \exp ^{2 \lambda t}} \\
b & =\sqrt{e_{2}^{2}(0)+e_{3}^{2}(0)}
\end{aligned}
$$

Let us start by demonstrating the global asymptotic stability of the rotational subsystem defined by (16) and (17). The subsystem formed by $e_{2}(t)$ and $e_{3}(t)$ is globally asymptotically stable if

$$
\lim _{t \rightarrow \infty} e_{2}(t)=0, \quad \lim _{t \rightarrow \infty} e_{3}(t)=0
$$


Both functions clearly tend to 0 when time approaches infinity since $\lim _{t \rightarrow \infty} a(t)=\infty$. Moreover, it is easy to show that $e_{2}(t)$ and $e_{3}(t)$ are strictly monotonic functions looking at their first derivative

$$
\dot{e}_{i}(t)=-\frac{2 \lambda e_{i}(0) \exp ^{2 \lambda t}\left(e_{2}^{2}(0)+e_{3}^{2}(0)+4\right)}{a(t)^{3}}
$$

with $i=\{2,3\}$. Note that the functions $e_{2}$ and $e_{3}$ are monotonic since the sign of their derivatives never changes and it only depends on the initial conditions. Furthermore, they are strictly monotonic since their derivative only zeroes when $t \rightarrow \infty$ or when the function at $t=0$ is already 0 . Therefore, for any initial condition, $e_{2}(t)$ and $e_{3}(t)$ always tend towards 0 strictly monotonically.

The global asymptotic stability of the translational subsystem depends on the behavior of $e_{1}(t)$. It is easy to show that $e_{1}(t)$ converges to 0 for any initial state since

$$
\left.\begin{array}{c}
\lim _{t \rightarrow \infty}\left(\frac{b(a(t)-2)}{b^{2}+2 a(t)}\right)=\frac{b}{2} \\
\lim _{t \rightarrow \infty} a(t)=\infty
\end{array}\right\} \Rightarrow \lim _{t \rightarrow \infty} e_{1}(t)=0
$$

In [11] it is shown that $e_{1}(t)$ is either strictly monotonic or it has an unique extremum before converging monotonically to 0 . In fact, a peak appears in $e_{1}(t)$ when the camera is initially very close to the desired distance and it is largely rotated with respect to the object [11].

We remember that given the control law (3) and the constant diagonal interaction matrix $\widehat{\mathbf{L}_{\mathrm{s}}}$ in (13) a proportional mapping from $\mathbf{e}$ to $\mathbf{v}$ is made. Therefore, the rotational velocities $\Omega_{x}$ and $\Omega_{y}$ converge strictly monotonically to 0 since $e_{2}(t)$ and $e_{3}(t)$ do. The camera orientation control can be considered as optimal and also the depth control when $e_{1}(t)$ is monotonic.

\section{MAKING FEATURES ROBUST AGAINST LASER-CROSS MISALIGNMENT}

During the modelling of our system we have assumed that the laser-cross can be perfectly aligned with the camera frame. However, this is not always possible because of the structure of the robot or because the optical center position is not exactly known. Therefore, it is important to study the robustness of the visual servoing approach against lasercross misalignment with respect to the camera. Unfortunately, simulations have shown that the set of visual features studied in the last section is quite unstable against small misalignment of the laser-cross for both control laws. We present now a simple method to enlarge the robustness domain of the features against laser-cross misalignment.

We are interested in defining a corrected set of visual features $\mathbf{s}^{\prime}$ which is analytically and experimentally robust against laser-cross misalignment. Fig. 2 shows the image point distribution in the desired state when different types of misalignment take place. As can be seen, a general misalignment of the laser-cross produces that the polygon enclosing the 4 points in the desired image appears misaligned and translated from the image center (indicated in Fig. 2 with a small cross).
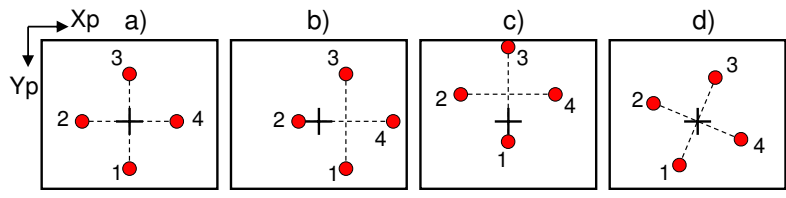

Fig. 2. Effects of laser-cross misalignment in the desired image. a) Ideal image. b) The laser-cross is horizontally displaced or rotated around ${ }^{C} Y$. c) The laser-cross is vertically displaced or rotated around ${ }^{C} Y$. d) Laser-cross rotated around ${ }^{C} Z$.

The idea consists of defining an image transformation which minimizes the misalignment observed in the image. This image transformation will be constrained as follows: in absence of laser-cross misalignment, the corrected set of visual features $\mathbf{s}^{\prime}$ must be equal to the uncorrected one $\mathbf{s}$. First of all, we eliminate the misalignment exhibited by the polygon in Fig. 2d which is produced when the laser-cross is rotated around the optical axis. Let us define the following unitary vectors

$$
\mathbf{x}_{42}^{*}=\left[\begin{array}{c}
x_{42}^{*} \\
y_{42}^{*}
\end{array}\right]=\frac{\mathbf{x}_{4}^{*}-\mathbf{x}_{2}^{*}}{\left\|\mathbf{x}_{4}^{*}-\mathbf{x}_{2}^{*}\right\|}, \mathbf{x}_{13}^{*}=\left[\begin{array}{c}
x_{13}^{*} \\
y_{13}^{*}
\end{array}\right]=\frac{\mathbf{x}_{1}^{*}-\mathbf{x}_{3}^{*}}{\left\|\mathbf{x}_{1}^{*}-\mathbf{x}_{3}^{*}\right\|}
$$

Then, a simple 2D transformation matrix of the form

$$
\mathbf{T}^{*}=\left[\begin{array}{ll}
\mathbf{x}_{24}^{*} & \mathbf{x}_{13}^{*}
\end{array}\right]^{-1}=\frac{1}{x_{42}^{*} y_{13}^{*}-x_{13}^{*} y_{42}^{*}}\left[\begin{array}{cc}
y_{13}^{*} & -x_{13}^{*} \\
-y_{42}^{*} & x_{42}^{*}
\end{array}\right]
$$

is defined so that $\mathbf{T}^{*}$ uses the desired image points in order to align the unitary vector corresponding to $\mathbf{x}_{4}-\mathbf{x}_{2}$ with the image axis $X_{p}$ and the unitary vector corresponding to $\mathbf{x}_{1}-\mathbf{x}_{3}$ with the image axis $Y_{p}$. Let us note the transformed image points as follows

$$
\mathbf{x}_{i}^{\prime \prime}=\mathbf{T}^{*} \mathbf{x}_{i}
$$

Then, it only rests to define a translation vector which is able to center the polygon in the image. The most intuitive choice is the center of gravity of the polygon $\mathbf{x}_{g}$. An expression of the center of gravity ensuring that $\mathbf{x}_{g}=\mathbf{0}$ when the lasercross is perfectly aligned is

$$
\mathbf{x}_{g}=\frac{1}{2}\left(\begin{array}{c}
x_{1}^{\prime \prime}+x_{3}^{\prime \prime} \\
y_{2}^{\prime \prime}+y_{4}^{\prime \prime}
\end{array}\right)
$$

Note that these expressions are 0 in the ideal case according to the image point coordinates in Table I. Then, the corrected image points are obtained as follows

$$
\mathbf{x}_{i}^{\prime}=\mathbf{x}_{i}^{\prime \prime}-\mathbf{x}_{g}=\mathbf{T}^{*} \mathbf{x}_{i}-\mathbf{x}_{g}
$$

And the corrected set of visual features $\mathbf{s}^{\prime}$ is therefore

$$
\mathbf{s}^{\prime}=\left(\begin{array}{lll}
y_{1}^{\prime}-1 & y_{3}^{\prime}-1 & y_{1}^{\prime}-1 \\
y_{3}^{\prime} & x_{2}^{\prime-1}+x_{4}^{\prime-1}
\end{array}\right)
$$

We remark that in the ideal case these corrected features are equal to the uncorrected ones, so that the global asymptotic stability under ideal conditions is also ensured. In addition to this, next section demonstrates that the corrected version is more robust against laser-cross misalignment. 


\section{STABILITY IN CASE OF LASER-CROSS MISALIGNMENT}

This section analyzes the stability of the system when using the corrected set of visual features $\mathbf{s}^{\prime}$ and when the lasercross frame $\{L\}$ is misaligned with respect to the camera frame $\{C\}$. This misaligment can be modelled by using a frame transformation formed by a rotation ${ }^{C} \mathbf{R}_{L}$ and a translation ${ }^{C} \mathbf{T}_{L}$. By using this frame transformation, all the parameters in Table I can be recalculated and consequently the real interaction matrix $\mathbf{L}_{\mathbf{s}^{\prime}}$. Finally, the closed-loop equation describing the dynamics of the task function including the misalignment can be obtained.

\section{A. Global asymptotic stability}

Unfortunately, in presence of misalignment errors the differential system (14) becomes strongly coupled so that the global asymptotic stability analysis is too complex. In order to simplify the problem we address the local asymptotic stability analysis around the desired state.

\section{B. Local asymptotic stability}

The local asymptotic stability analysis consists of linearizing the closed-loop equation of the system (14) around the desired state

$$
\dot{\mathbf{e}}=-\lambda \mathbf{L}_{\mathbf{s}^{\prime}}^{*} \widehat{\mathbf{L}_{\mathbf{s}^{\prime}}}+\mathbf{e}
$$

where $\mathbf{L}_{\mathbf{s}^{\prime}}^{*}$ is the interaction matrix containing the misalignment parameters and evaluated in the desired state. The computation of $\mathbf{L}_{\mathbf{s}^{\prime}}^{*}$ for different relative camera-lasers poses is presented in [11]. On the other hand, $\widehat{\mathbf{L}_{\mathbf{s}^{\prime}}}$ is the interaction matrix used in the control law which is based on the ideal model and evaluated in the desired state. Therefore, for any of the two control laws $\widehat{\mathrm{L}_{\mathrm{s}^{\prime}}}$ is the diagonal matrix in (13).

The system is said to be locally asymptotically stable if and only if the eigenvalues of the matrix $\mathbf{M}=\mathbf{L}_{\mathbf{s}^{\prime}}^{*} \widehat{\mathbf{L}}_{\mathbf{s}}^{+}$have all positive real part. The eigenvalues calculation is too complex if a whole model of misalignment is applied. We first consider that the laser-cross is displaced from the camera origin but it preserves the same orientation that the camera frame, i.e. ${ }^{C} \mathbf{T}_{L}=\left(t_{x}, t_{y}, t_{z}\right)$ and ${ }^{C} \mathbf{R}_{L}=\mathbf{I}_{3}$. In this case, one can show that the eigenvalues are all equal to 1 so that the local asymptotic stability in front of this type of misalignment is ensured. On the other hand, when the laser-cross is rotated with respect to any one of the camera axis, the eigenvalues are always positive so that the local asymptotic stability against this type of misalignment is also ensured.

The proof that the set of visual features $\mathbf{s}^{\prime}$ is more robust against laser-cross misalignment is clear if we develop the local asymptotic stability analysis of the set $\mathbf{s}$. For $\mathbf{s}$, when the laser-cross is displaced, the positivity of the eigenvalues is only ensured when

$$
\left|t_{x}\right|<L, \quad\left|t_{y}\right|<L
$$

And for the case of a rotated laser-cross, the positivity of the eigenvalues is neither ensured [11]. Therefore, the convergence domain in presence of laser-cross misalignment is actually larger with the corrected set of visual features.

\section{EXPERIMENTAL RESULTS}

In this section we present experimental results which show the performance of the corrected set of visual features $\mathbf{s}^{\prime}$. The experimental setup is based on a six dof robot with a $8.5 \mathrm{~mm}$ focal length camera coupled to the end-effector. The lasercross was built so that $L=15 \mathrm{~cm}$. The desired position was defined so that the camera is at $60 \mathrm{~cm}$ parallel to a plane situated in front of the robot.

In the first experiment, the laser-cross was approximately aligned with the camera frame. The initial position was defined by moving the robot backwards $45 \mathrm{~cm}$ from the desired position and rotating -20 and 20 degrees around the $X$ and $Y$ axis, respectively. The initial and desired images of the experiment are shown in Fig. 3a-b. We first note that the image point trajectories shown in the desired image follow straight lines as explained in [10]. On the other hand, since these trajectories are not perfectly aligned with the image axis it means that the laser-cross and the camera are slightly misaligned. When using the non-constant control law, the task function response is shown in Fig. $3 \mathrm{c}$ and the camera velocities are plotted in Fig. 3d. The task function has not a pure exponential decrease as expected in the ideal case due to the calibration errors. Note that the behavior of the camera velocities is strongly non-monotonic. On the other hand, when using the constant control law, the analytic model predicts that both the task function and the camera velocities should be monotonic under ideal conditions. In Fig. 3e-f the task function and camera velocities obtained in the experiment using the constant control law are shown. Note that both the task function and the camera velocities follow the model prediction.

The aim of the second experiment was to check the robustness of the system in front of a large misalignment of the laser-cross. The laser-cross was displaced about $6 \mathrm{~cm}$ along the $X$ camera axis, rotated about $7^{\circ}$ around the $Z$ axis and smaller rotations were applied around $X$ and $Y$. The large misalignment is clearly observed in the initial and desired images shown in Fig. 4a-b. With such a large misalignment, the non-constant control law has failed for different initial positions because the robot has reached a joint limit. Actually, the non-monotonic behavior of the camera velocities is stronger due to the large calibration errors, producing some joint motions which are unfeasible. On the other hand, when using the constant control law the camera reaches the desired position with no problems. The task function still converges almost monotonically to 0 as shown in Fig. 4c. Concretely, the visual feature controlling the depth is unaffected by the misalignment, while the monotonic decrease of the features controlling the rotational part is slightly affected. Note that the camera velocities shown in Fig. 4d still have a nice decrease to 0 which implies that the camera trajectory is only slightly affected by the large misalignment of the laser-cross.

\section{CONCLUSIONS}

This paper presents an image-based visual servoing approach based on structured lighting for plane-to-plane po- 
a)

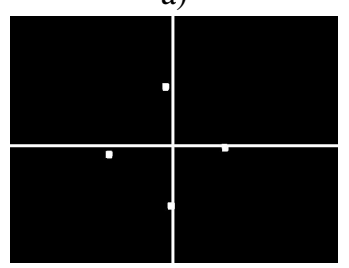

c)

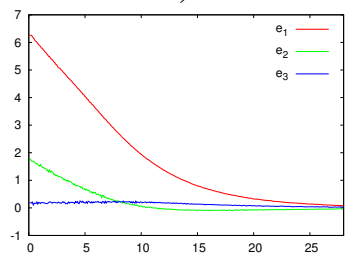

e)

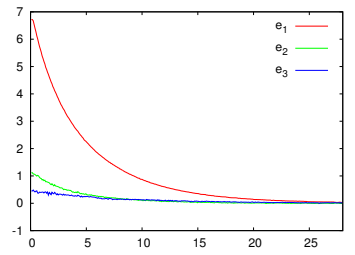

b)

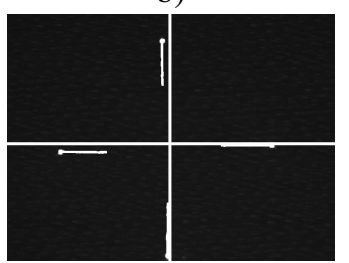

d)

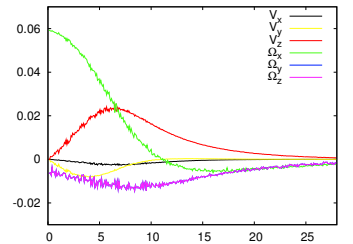

f)

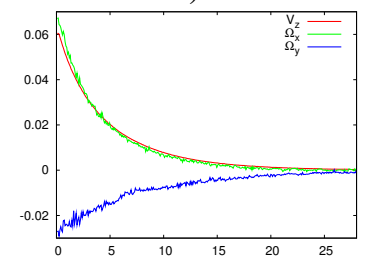

Fig. 3. Experimental results. Coarse alignment of the laser-cross: a) Initial camera image including image axis. b) Laser points trajectory from initial to desired position. c-d) Results using the non-constant control law. e-f) Results using the constant control law. c) and e) show the task function vs. time (in seconds). $d$ and f) show the camera velocities vs. time in $\mathrm{m} / \mathrm{s}$ and $\mathrm{rad} / \mathrm{s}$.

sitioning. The paper presents a new set of 3 visual features which decouples rotational from translational dof in the entire workspace. Thanks to the decoupled form of the general interaction matrix, we have succeeded in proving that, in absence of calibration errors, the system converges to the desired state for any initial camera-object pose when using two different control laws. The first control law uses an estimation of the interaction matrix at each iteration and the second one uses the interaction matrix evaluated in the desired state. In both cases we have succeed on demonstrating that the task function converges monotonically to 0 . The paper also presents a simple feature transformation which enlarges the convergence domain in presence of large misalignments between the camera and the laser-cross. Real experiments have been carried out in order to validate the analytic results. They clearly show that the task function has a nice decrease for both control laws even in presence of large misalignment of the laser-cross. However, the non-constant control law produces non-monotonic velocities which under large calibration errors may become unfeasible. On the other hand, the constant control law has shown better performance since it provides a linear mapping from the task function space to the camera velocities so that the latter are also monotonic even in presence of large calibration errors.

\section{ACKNOWLEDGments}

Work partially funded by the Cemagref Rennes of France, the OFIVAL and the Ministry of Universities, Research and a)

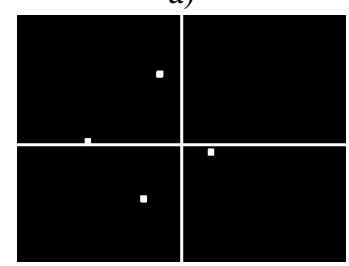

c)

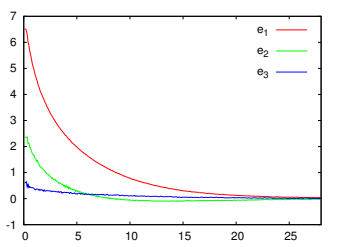

b)

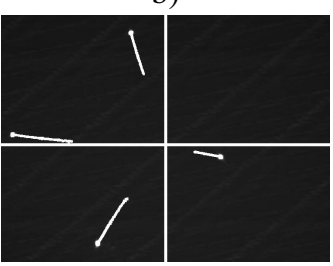

d)

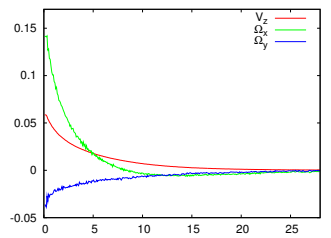

Fig. 4. Experiment with large laser-cross misalignment. a) Initial camera image. b) Laser points trajectory from the initial to desired position. c) Task function vs. time (in seconds). d) Camera velocities vs. time (m/s and $\mathrm{rad} / \mathrm{s}$ ).

Information Society of the Catalan Government.

\section{REFERENCES}

[1] N. Andreff, B. Espiau, and R. Horaud. Visual servoing from lines. Int. Journal of Robotics Research, 21(8):679-700, August 2002.

[2] P. I. Corke and S. A. Hutchinson. A new partitioned approach to imagebased visual servo control. IEEE Trans. on Robotics and Automation, 17(4):507-515, August 2001.

[3] K. Deguchi. Direct interpretation of dynamic images and camera motion for visual servoing without image feature correspondence. Journal of Robotics and Mechatronics, 9(2):104-110, 1997.

[4] B. Espiau, F. Chaumette, and P. Rives. A new approach to visual servoing in robotics. IEEE Trans. on Robotics and Automation, 8(6):313-326, June 1992.

[5] S. Hutchinson, G. Hager, and P. Corke. A tutorial on visual servo control. IEEE Trans. on Robotics and Automation, 12(5):651-670, 1996.

[6] D. Khadraoui, G. Motyl, P. Martinet, J. Gallice, and F. Chaumette. Visual servoing in robotics scheme using a camera/laser-stripe sensor. IEEE Trans. on Robotics and Automation, 12(5):743-750, 1996.

[7] A. Krupa, J. Gangloff, C. Doignon, M. Mathelin, G. Morel, J. Leroy, L. Soler, and J. Marescaux. Autonomous 3d positionning of surgical instruments in robotized laparoscopic surgery using visual servoing. IEEE Trans. on Robotics and Automation, 19(5):842-853, 2003.

[8] R. Mahony, P. Corke, and F. Chaumette. Choice of image features for depth-axis control in image-based visual servo control. In IEEE/RSJ Int. Conf. on Intellingent Robots and Systems, Lausanne, Switzerland, September 2002.

[9] E. Malis and F. Chaumette. Theoretical improvements in the stability analysis of a new class of model-free visual servoing methods. IEEE Trans. on Robotics and Automation, 18(2):176-186, April 2002.

[10] J. Pages, C. Collewet, F. Chaumette, and J. Salvi. Plane-to-plane positioning from image-based visual servoing and structured light. In IEEE/RSJ Int. Conf. on Intelligent Robots and Systems, volume 1, pages 1004-1009, Sendai, Japan, September 2004.

[11] J. Pagès, C. Collewet, F. Chaumette, and J. Salvi. Visual servoing by means of structured light for plane-to-plane positioning. Technical Report 5579, INRIA, May 2005.

[12] F. Schramm, G. Morel, A. Micaelli, and A. Lottin. Extended-2d visual servoing. In IEEE Int. Conf. on Robotics and Automation, pages 267273, New Orleans, USA, April 26-May 12004.

[13] O. Tahri and F. Chaumette. Image moments: Generic descriptors for decoupled image-based visual servo. In IEEE Int. Conf. on Robotics and Automation, volume 2, pages 1185-1190, New Orleans, LA, April 2004. 